\title{
The effects of neuromonitorization in thyroidectomies can be safely evaluated with the standardized technique
}

\author{
(D) Mert Tanal, (D) Mehmet Uludag \\ Department of General Surgery, University of Health Sciences, Istanbul Sisli Hamidiye Etfal Health Training and Research Hospital, \\ Istanbul, Turkey
}

Cite this article as: Tanal M, Uludag M. The effects of neuromonitorization in thyroidectomies can be safely evaluated with the standardized technique. North Clin Istanb 2018;5(3):274-276.

To the Editor,

We read the article written by Demiryas et al. [1] with interest, and we would like to offer some observations about the study based on the current literature.

Though the purpose of the study was to determine the effect of intraoperative neuromonitoring (IONM) on thyroid surgery complications, the explanation of the IONM technique given in the Materials and Methods section was very limited. In addition, it would appear that only the recurrent laryngeal nerve (RLN) was stimulated and vagus nerve (VN) stimulation was not performed. The International Neural Monitoring Study Group published guidelines for RLN monitoring during thyroid surgery in 2011. The standardized technique includes VN stimulation both before thyroid dissection (V1) and after complete thyroidectomy (V2) [2]. V1 is used as a reference to verify the function of the IONM system and allows for subsequent dissection and RLN identification. $\mathrm{V} 2$ testing is the most appropriate technique to predict postoperative vocal cord function [2].
This protocol must be applied in order to optimize the prognostic value of IONM [2]. We suggest that because this standardized technique was not used, and because this study includes the data of patients from 2014 to 2016, the results regarding the effects of IONM might be misleading.

Near-total thyroidectomies in which the RLN is partially visualized and total thyroidectomies in which the RLN is totally visualized were included in both groups. These two different techniques may lead to different RLN paralysis results. Complete dissection is greatly superior to simply localized or partial exposure of the nerve [3]. What were the vocal cord paralysis ratios of the near-total and total thyroidectomies?

The preoperative diagnosis and hyperthyroidism ratios for the two groups were not provided. A high-risk thyroidectomy case, such as a substernal goiter, thyroid cancer, or Graves disease, may prolong the operation time. Without evaluating these factors, can the short duration of the operations in Group 1 only be attributed to IONM? 
The total hypocalcemia ratios were lower in the IONM group. Surgical factors, such as the extent of surgery, central node dissection, reoperation for bleeding, presence of Graves disease or thyroid cancer, operation for recurrent goiter, inadvertent parathyroid excision, and parathyroid autotransplantation may also affect the postoperative ratios of hypocalcemia [4]. Can a reduction in the rate of hypocalcemia be attributed to IONM without assessing these considerations?

The conclusion of the article mentions that the authors' research and similar studies in the literature did not reveal a benefit to IONM usage in terms of decreasing the rate of RLN injury. In the literature, the impact of IONM on RLN paralysis is still controversial. Nonetheless, in a recent meta-analysis of 34 comparative studies on this subject, it was determined that IONM significantly reduced total, temporary, and permanent RLN paralysis [5].

This study [1] is a retrospective study. Features such as the preoperative diagnosis, which can affect the results, were not evaluated and compared. The number of cases was insufficient for a reliable assessment and the standard IONM technique was not implemented. Considering all of these limitations, the conclusions about IONM are questionable.

\section{REFERENCES}

1. Demiryas S, Donmez T, Cekic E. Effect of nerve monitoring on complications of thyroid surgery. North Clin Istanb 2018;5:14-9.

2. Randolph GW, Dralle $\mathrm{H}$; International Intraoperative Monitoring Study Group, Abdullah H, Barczynski M, Bellantone R, et al. Electrophysiologic recurrent laryngeal nerve monitoring during thyroid and parathyroid surgery: international standards guideline statement. Laryngoscope 2011;121 Suppl 1:S1-16. [CrossRef]

3. Hermann M, Alk G, Roka R, Glaser K, Freissmuth M. Laryngeal recurrent nerve injury in surgery for benign thyroid diseases: effect of nerve dissection and impact of individual surgeon in more than 27,000 nerves at risk. Ann Surg 2002;235:261-8. [CrossRef]

4. Lorente-Poch L, Sancho JJ, Muñoz-Nova JL, Sánchez-Velázquez P, Sitges-Serra A. Defining the syndromes of parathyroid failure after total thyroidectomy. Gland Surg 2015;4:82-90.

5. Bai B, Chen W. Protective Effects of Intraoperative Nerve Monitoring (IONM) for Recurrent Laryngeal Nerve Injury in Thyroidectomy: Meta-analysis. Sci Rep 2018;8:7761. [CrossRef]

\section{AUthoR'S REPLY}

To the Editor,

We are grateful for the interest of our colleagues in our article, "Effect of nerve monitoring on complications of thyroid surgery," published in the Northern Clinics of Istanbul journal. However, we strongly believe that the stated aim and context of our article was not fully appreciated and that our clearly written purpose was not given enough attention. As we explained, it was a retrospective study of thyroid surgeries performed between 2014 and 2016 at a single center by a single surgeon. Our research was accepted for publication in 2017; therefore, data from the mentioned review article from 2018 was not available for consideration for this paper.

Our study included a group of patients who had thyroidectomy indications and the operations were approved by an anesthesiology specialist at our state hospital. All of these patients were in a euthyroid state, and their postoperative primary pathological diagnoses were also reported in our published paper. Preoperative diagnoses were disregarded in order to preserve the reliability and focus of our article, since there was no significant statistical correlation between the pathological primary diagnoses and the complications encountered. Interventions such as parathyroid surgery, retrosternal goiter surgery, preoperative planned oncological surgery, or neck dissection did not need to be mentioned because additional procedures were not performed at that time. The postoperative primary pathological diagnoses were reported; however, we did not mention each patient's pathological diagnosis in greater detail in order to avoid adding unnecessary data. Furthermore, if the inclusion of the details mentioned above would have made an additional contribution to our paper, the editorial experts would certainly have urged us to do so during the meticulous evaluation period. As our colleagues will surely agree, when the text of an article is lengthened with nonessential information the reader may be distracted from the focus of article and the intended message may be lost. 